\title{
Variaciones en los años de vida perdidos por cánceres de mama y cérvico uterino en México según grado de marginación estatal, 1997 y 2007
}

\author{
MARCELA AGUDELO $^{(1)}$, ALEJANDRO AGUIRRE(1) y CLAUDIO DÁVILA ${ }^{(1)}$
}

\section{RESUMEN}

Objetivo: Calcular los Años de Vida Perdidos (AVP) por cáncer de mama (CaMa) y cérvico uterino (CaCu) en México para 1997 y 2007, de acuerdo con el índice de marginalidad estatal. Material y Método: Estudio de tipo descriptivo, transversal y comparativo en donde se utilizaron las Estadísticas Vitales de Mortalidad para CaMa y CaCu a nivel nacional y por grupos quinquenales de edad (entre los 20 y 79 años). Para la estimación de los AVP se empleó la técnica propuesta por Arriaga en 1996, en la cual se maneja un supuesto de mortalidad nula entre las edades analizadas. Por último, se usó la estratificación por grado de marginación estatal, elaborada por el Consejo Nacional de Población (CONAPO) en 2005. Resultados: Se observó una disminución de los AVP por CaCu (0.048) y un aumento de los mismos por CaMa (0.016) en el decenio analizado. Por conglomerados se advirtió que las mujeres que vivían en zonas con índice de marginación alto y muy alto tuvieron más AVP debido al CaCu y las mujeres de áreas más desarrolladas fueron las que más AVP por CaMa registraron; el grupo de marginación medio tuvo el mayor decremento en los AVP por CaCu y el crecimiento más marcado de AVP por CaMa, frente a los otros dos grupos en comparación. Discusión: Tanto las tendencias observadas para $\mathrm{CaCu}$ y CaMa como las diferencias por grado de marginación estatal se asemejan a los resultados obtenidos en otros contextos latinoamericanos.

Palabras clave: cáncer de mama, cáncer cérvico-uterino, años de vida perdidos, índice de marginalidad.

\section{ABSTRACT}

VARIATION IN LIFE YEARS LOST FROM BREAST AND CERVICAL CANCER BY NATIONAL MARGINALIZATION INDEX IN MEXICO, 1997 TO 2007

Objective: Calculate the Years of Life Lost (YLL) for breast cancer (BC) and cervical cancer (CC) in Mexico from 1997 to 2007, by the national marginalization index. Materials and Methods: Descriptive, cross sectional and comparative study using mortality data from national vital statistics for $B C$ and $C C$ by five year age groups (between 20 and 79 years). To estimate the YLL, the technique proposed by Arraiga in 1996 was used, assuming no mortality in the age groups analyzed. Data was stratified by level of marginalization, according to the National Population Council (CONAPO) in 2005. Results: A decrease in YLL for CC (0.048) and an increase for BC were observed in the decade analyzed. Women who live in areas with a high or very high marginalization index had more YLL due to CC, and women in more developed areas had the highest YLL for BC.

(1) El Colegio de México. Camino al Ajusco No. 20. Pedregal de Santa Teresa, Magdalena Contreras. Código Postal 10740. México. Distrito Federal.magudelo@colmex.mx 
The middle marginalization group had the greatest decrease in YLL due to CC and the biggest increase in YLL due to BC, compared to the other two groups. Discussion: Both the observed tendencies for $C C$ and $B C$ and the differences according to level of marginalization resemble results obtained in other contexts in Latin America.

Keywords: breast cancer, cervical cancer, years of life lost, marginalization index.

\section{INTRODUCCIÓN}

Los análisis de los niveles y tendencias de la mortalidad se han convertido en instrumentos fundamentales para la planificación y establecimiento de políticas gubernamentales en diversos campos, al tiempo que han permitido poner en relieve la heterogeneidad de condiciones de vida entre distintas poblaciones ${ }^{1}$. El fenómeno de la mortalidad es un indicador sensible que pone de manifiesto las características generales de los individuos, especialmente en lo que respecta al ámbito socioeconómico y sanitario ${ }^{2,3}$.

Si bien es cierto que en la actualidad existen múltiples técnicas de medición de la mortalidad (tasas brutas, tasas específicas, esperanza de vida al nacimiento), ninguna tiene la capacidad de reflejar de manera sintética los aspectos relacionados con el nivel y el cambio de la misma ${ }^{4-6}$. En México, la mayoría de los estudios (tanto epidemiológicos como demográficos) se ha basado principalmente en los indicadores antes mencionados para dar cuenta del panorama actual frente a la mortalidad, reflejando que el país ha ido experimentando a lo largo de los últimos tiempos constantes cambios en los patrones y número de fallecimientos, pasando de un escenario donde se observa una disminución de muertes de índole transmisible y un aumento progresivo de muertes de tipo crónico-degenerativas ${ }^{7,8}$.

A grandes rasgos se resalta que para 1997 el total de muertes notificadas en el país fue de 440.437 , las cuales se concentraban principalmente en tres causas: enfermedades del corazón, tumores malignos y diabetes mellitus, patologías que en su conjunto representaron $43.5 \%$ de las muertes. Diez años más tarde, es decir para 2007, las enfermedades del corazón continuaron ocupando el primer lugar como causa de muerte, seguido por diabetes y tumores malignos, las cuales agruparon $50.4 \%$ del total de defunciones registradas en este año $(514.420)^{9}$.

En 2007 los tumores malignos representaron $14.6 \%$ de los fallecimientos femeninos versus $11.1 \%$ entre los varones. Dentro de las neoplasias malignas entre mujeres, el cáncer de mama $(\mathrm{CaMa})$ y el cáncer cérvico uterino $(\mathrm{CaCu})$ fueron las dos causas que mayor registro de muertes tuvieron para el grupo femenino de 25 años y más ${ }^{9}$. Históricamente estas dos afecciones han punteado la lista como primeras causa de muerte por tumores en este sexo y para este rango de edad, aunque es a partir de 2006 que el CaMa sobrepasa en registros el $\mathrm{CaCu}$, situación que se mantiene hasta la fecha ${ }^{10}$.

Sobre estos dos padecimientos en particular, se han realizado abundantes estudios que develan que los principales problemas respecto de estas causas de muerte se centran alrededor de barreras de acceso y calidad de atención en los servicios de salud. Asimismo, se ha encontrado una polarización de los niveles de mortalidad de acuerdo con el grado de desarrollo socioeconómico y otras características geográficas en México, por lo cual se considera pertinente incorporar esta variable en el estudio de la mortalidad por $\mathrm{CaCu}$ y $\mathrm{CaMa}^{11-15}$.

En este trabajo se tiene como objetivo principal contribuir al conocimiento del impacto de la mortalidad por $\mathrm{CaMa}$ y $\mathrm{CaCu}$ en el territorio mexicano según el grado de marginación estatal. Para ello se emplea el cálculo de Años de Vida Perdidos (AVP)a y se

a No debe confundirse con los Años de Vida Potencialmente Perdidos (AVPP) utilizado frecuentemente por la Organización Panamericana de la Salud (OPS). 
comparan dos momentos en el tiempo (1997 y 2007), con el fin de observar los cambios acaecidos en dicho periodo.

Los AVP son un indicador con el cual se trata de determinar cuántos años de vida en promedio pierde una población por la muerte de las personas a determinadas edades y por diferentes causas. Esta técnica ha sido poco empleada en los estudios de mortalidad en México, a pesar de que es una de las principales herramientas para medir los cambios en el nivel de la mortalidad ${ }^{4,5}$.

\section{MATERIAL Y MÉTODO}

Estudio de tipo descriptivo, transversal y comparativo en donde se utilizaron las estadísticas vitales de mortalidad a nivel nacional para 1997 y 2007 provenientes del Instituto Nacional de Estadística, Geografía $(\mathrm{INEGI})^{9}$. Se realizaron cálculos generales de la mortalidad femenina, así como para $\mathrm{CaMa}$ y $\mathrm{CaCu}$, por grupos quinquenales de edad (a partir de los 20 años y concluyendo con el grupo de 75-79 años). Se homogenizaron los datos por CaMa y $\mathrm{CaCu}$ en los dos años ya mencionados según los criterios de Clasificación Internacional de Enfermedades $(\mathrm{CIE})^{\mathrm{b}}$, en su novena y décima revisión ${ }^{16,17}$. Por otra parte, fue necesario hacer las siguientes acotaciones metodológicas:

- Se excluyeron de la base de datos aquellos registros no especificados por edad y sexo.

- No fueron consideradas aquellas muertes ocurridas en el exterior.

- Dado que se calcularon años de vida perdidos entre los 20 y los 79 años, se descartaron los casos de muerte acaecidos en mujeres menores de 20 años y de 80 años en adelante.

- Se omitieron de la investigación las notificaciones de muerte por CaMa entre varones.
El total de registros por CaMa y $\mathrm{CaCu}$ fue de 7.754 para 1997 , de los cuales se descartaron $10.7 \%$ de individuos; en 2007 fue de 8.694 y se exceptuaron $11.6 \%$ de casos para este mismo año.

\section{Estimación de los años de vida perdidos}

Se calcularon los AVP propuestos por Arriaga (1996) 4,5 los cuales tratan de determinar cuántos años de vida en promedio pierde una población por muerte a determinadas edades. Para ello, se parte del supuesto de que la mortalidad debería haber sido nula entre dos edades elegidas para el análisis. Para esta investigación se consideró como límites de edad los 20 y los 79 años de edad. Lo que subyace en el fondo de esta técnica es que los que murieron deberían haber vivido hasta la edad superior del intervalo de edades dentro del que se analiza la mortalidad ${ }^{4,5}$. El cálculo de los AVP parte de los resultados de tablas de mortalidad en lo relacionado con las defunciones y los sobrevivientes; En este estudio se emplearon las tablas de vida femenina del año 2001 propuestas por Mina ${ }^{18}$.

El primer paso propuesto por Arriaga ${ }^{4,5}$ es suponer que las defunciones por causa de muerte de la tabla de mortalidad abreviada en cada grupo de edad guardan la misma distribución

$$
{ }_{n} d_{x, j}={ }_{n} d_{x}\left(\frac{{ }_{n} D_{x, j}}{{ }_{n} D_{x}}\right)
$$

que las defunciones observadas, entonces:

Los que mueren en el grupo de edad $\mathrm{x} \mathrm{a} \mathrm{x}+\mathrm{n}$ pierden tantos años de vida dentro de ese grupo de edad como el producto de las defunciones de la causa de muerte por la diferencia entre el intervalo del grupo de edad $\mathrm{n}$ y el factor de separación de las defunciones $\mathrm{n}_{\mathrm{n}}$. Si se utiliza el supuesto de distribución uniforme de muerte entre las edades $\mathrm{x} \mathrm{y} \mathrm{x}+\mathrm{n}$, el factor de separación es igual a 2.5. Los años AVP por aquellos que

b De acuerdo con la CIE-9 corresponde a los códigos 174 y 180 el CaMa y el CaCu respectivamente (aplicado para 1997), mientras que para la CIE-10 (que abarca el 2007) los códigos de estas causas fueron C50 para CaMa y C53 para $\mathrm{CaCu}$. 
fallecen por la causa de muerte $\mathrm{j}$ en las edades de $\mathrm{x}$ a $\mathrm{x}+\mathrm{n}$ años son:

$$
2{ }_{\mathrm{u}, \mathrm{n}} \mathrm{AP}_{\mathrm{x}, \mathrm{j}}=\mathrm{n}_{\mathrm{n}, \mathrm{j}}\left[\left(\mathrm{n}-\mathrm{n}_{\mathrm{n}} \mathrm{k}_{\mathrm{x}}\right)+(\mathrm{v}-\mathrm{x}-\mathrm{n})\right]
$$

Asimismo, el promedio de AVP por las personas que están vivas a la edad inicial al intervalo de edades seleccionadas, debido a la mortalidad por la causa $\mathrm{j}$ (en este estudio $\mathrm{CaMa}$ o $\mathrm{CaCu}$ ) en el grupo de edad $\mathrm{x}$ a $\mathrm{x}+\mathrm{n}$ y se obtiene de:

3) ${ }_{u, n} a p_{x, j}=\frac{\left[{ }_{n} d_{x, j}\left(v-{ }_{n} k_{x}-x\right)\right]}{1_{a}}$

donde la es el número de personas vivas a la edad exacta a en la tabla de mortalidad.

La suma de los AVP entre las edades a y v da el promedio de AVP por la causa de muerte $\mathrm{j}$ en el intervalo de edad de u años:

$$
\mathrm{ap}_{\mathrm{j}, \mathrm{j}}=\sum_{x=a}^{v} \mathrm{u}, \mathrm{n}_{\mathrm{x}, \mathrm{j}}
$$

\section{Análisis estratificado}

Otra variante del análisis consistió en indicar las diferencias socioeconómicas en la mortalidad por CaMa y $\mathrm{CaCu}$, en términos de los AVP y los patrones de cambios a los que hubo lugar entre un año y otro. Para ello se utilizó el índice de marginalidad ${ }^{c}$ propuesto por el Consejo Nacional de Población (CONAPO) ${ }^{19}$ en 2005. La tipificación hecha por CONAPO contempla 5 grupos de marginación (muy alta, alta, media, baja y muy baja), sin embargo, para facilitar el manejo de la información y el contraste entre conglomerados, se eligió hacer una reagrupación, quedando al final sólo 3 grupos de observación (Tabla 1).

\section{Herramientas de análisis}

Se empleó el programa Statistical Package for the Social Sciences (SPSS) en su versión 17.0 para el manejo de las estadísticas vitales de mortalidad y también se usaron tablas de excel para la construcción del indicador de AVP basado en tablas de vida.

\section{RESULTADOS}

\section{Análisis por causas de mortalidad y grupos de edad}

Para 1997 los AVP por $\mathrm{CaCu}$ eran superiores que los AVP por CaMa, situación que se invirtió para 2007. En el periodo analizado hubo un incremento en el promedio de AVP por $\mathrm{CaMa}$ de 0.016 y una disminución de 0.048 por $\mathrm{CaCu}$ (ver tabla 2). En cuanto al número de defunciones registradas, en 1997 ocurrieron $40.6 \%$ de las muertes por CaMa en mujeres menores de 50 años, mientras que para 2007 las muertes en este grupo etario representaron $34.9 \%$. En el caso del $\mathrm{CaCu}$ no se evidenciaron cambios sustanciales, la distribución porcentual de la mortalidad en estos dos años fue de $35.5 \%$ y $32.2 \%$, para 1997 y 2007 respectivamente.

Vale la pena resaltar que sólo los tres primeros grupos de edad de este estudio redujeron sus AVP debido al CaMa y las mujeres de 20-24 años fueron las únicas que aumentaron los AVP como consecuencia del $\mathrm{CaCu}$. Otro aspecto importante es que en todos los años y para las dos causas, las mujeres del grupo de edad de 50-54 años fueron las que más años de vida perdieron con respecto a las demás mujeres. Junto con estas últimas, las mujeres entre 5559 años tuvieron el mayor aumento en el promedio de AVP por CaMa (0.004 para cada grupo); se advirtió también un decremento significativo para $\mathrm{CaCu}$ en el grupo de 70-74 años (0.008).

\section{Diferencias socioeconómicas en los años de vida perdidos}

Se hizo una categorización de los 32 Estados que conforman la República basada en el impacto global de las privaciones que padece la población, como resultado de la falta de acceso a la educación y la salud, la residencia en viviendas inadecuadas y la carencia de bienes de primera necesidad. 
Tabla 1. Grado de marginación por entidad federativa. México, 2005.

\begin{tabular}{|l|l|}
\hline Grado de marginación & \multicolumn{1}{c|}{ Estados } \\
\hline Muy alto y alto & $\begin{array}{l}\text { Oaxaca, Chiapas Guerrero, Yucatán, Tabasco, Puebla, Hidalgo, Veracruz, } \\
\text { San Luis Potosí, Michoacán, Campeche }\end{array}$ \\
\hline Medio & Zacatecas, Tlaxcala, Nayarit, Guanajuato, Durango, Sinaloa, Querétaro \\
\hline Muy bajo y bajo & $\begin{array}{l}\text { Quintana Roo, México, Aguascalientes, Morelos, Sonora, Colima, Tamau- } \\
\text { lipas, Chihuahua, Jalisco, Baja California Sur, Baja California, Coahuila, } \\
\text { Nuevo León, Distrito Federal }\end{array}$ \\
\hline
\end{tabular}

Fuente: Elaboración propia a partir de información del CONAPO, 2005.

En el análisis estratificado de los AVP por CaMa y $\mathrm{CaCu}$ se evidenciaron cambios sustantivos y relevantes. Por un lado, se muestra que entre 1997 y 2007 se dio un incremento en el promedio de AVP por CaMa para todos los conglomerados, siendo más acentuado en el de mediana marginación; También se observa una reducción constante en el promedio de AVP por $\mathrm{CaCu}$, igualmente más marcado para las mujeres de áreas con marginación media (Figuras 1 y 2). Cabe señalar que para 2007, 24.8\% de las muertes por $\mathrm{CaMa}$ acontecieron en estados con marginalidad alta y muy alta, mientras que un $40.4 \%$ de las defunciones por $\mathrm{CaCu}$ se presentaron en dichos lugares. Esta situación fue diferente a la que ocurrió en las zonas de marginalidad baja y muy baja donde sucedieron $39.5 \%$ de las defunciones por CaMa y $30.0 \%$ de las defunciones por $\mathrm{CaCu}$; entre tanto, en zonas de marginación media se concentró un $35.7 \%$ de las muertes por CaMa y el $29.6 \%$ por $\mathrm{CaCu}$.

Existen contrastes inversos en el promedio de AVP para CaMa y $\mathrm{CaCu}$ : en el primer caso, hubo mayor pérdida de años de vida para mujeres con bajo rezago socioeconómico y se perdieron menos años de vida en zonas con alta y muy alta marginalidad (tanto para 1997 como para 2007). Por el contrario, las mujeres más pobres tuvieron promedios de AVP por $\mathrm{CaCu}$ superiores a sus homólogas con índices de marginación bajo. Los aumentos de los AVP por CaMa, entre un año y otro, fueron moderados para todos los conglomerados con distintos grados de marginación, en cambio, para el $\mathrm{CaCu}$ se notan reducciones más marcadas en los AVP; incluso para 2007 se percata que el promedio de AVP por $\mathrm{CaCu}$ es casi igual para los estados con marginación media y la más baja.

Finalmente, para todos los estratos, el promedio de AVP por $\mathrm{CaCu}$ en 1997 superaba considerablemente el promedio de AVP por CaMa. Sin embargo, para 2007 las mujeres de regiones con marginalidad media, muy baja y baja perdieron más años de vida por CaMa que por $\mathrm{CaCu}$. Aunque el $\mathrm{CaCu}$ sigue ubicándose por encima del CaMa en áreas de marginalidad alta y muy alta, estas cifras se han acercado cada vez más, debido a la notoria disminución de las defunciones por $\mathrm{CaCu}$ y un aumento sostenido del CaMa.

\section{DISCUSIÓN}

Enfermedades como el CaMa y el CaCu traen graves consecuencias para la salud y vida de las mexicanas ${ }^{11-13,20,21}$. De acuerdo con cifras arrojadas en este trabajo, poco más de $1 / 3$ de las muertes por estas neoplasias se presenta a edades tempranas, en este caso antes de los 50 años. El hecho de que el fallecimiento se produzca a edades jóvenes (algo muy diferente a lo que ocurre generalmente con otras enfermedades crónico-degenerativas $)^{10}$, requiere que su detección y tratamiento llegue oportunamente y, al mismo tiempo, se utilicen estrategias de atención que cubran las diferentes necesidades y demandas en la materia ${ }^{11,13,20}$. Estos mismos argumentos se han utilizado en otros estudios donde se enfatiza la importancia de 
Tabla Defunciones, años de vida perdidos y promedio de años de vida perdidos por cánceres de

2. mama y cérvico uterino según grupos quinquenales de edad. México, 1997 y 2007.

\begin{tabular}{|c|c|c|c|c|c|c|}
\hline \multicolumn{7}{|c|}{ Cáncer de mama } \\
\hline \multirow{2}{*}{$\begin{array}{l}\text { Grupo } \\
\text { de edad }\end{array}$} & \multicolumn{3}{|c|}{1997} & \multicolumn{3}{|c|}{2007} \\
\hline & Defunciones & AVP & $\begin{array}{c}\text { Promedio } \\
\text { de AVP }\end{array}$ & Defunciones & AVP & $\begin{array}{c}\text { Promedio } \\
\text { de AVP }\end{array}$ \\
\hline $20-24$ & 8 & 27 & 0.000 & 7 & 21 & 0.000 \\
\hline $25-29$ & 44 & 164 & 0.002 & 39 & 131 & 0.001 \\
\hline $30-34$ & 125 & 472 & 0.005 & 119 & 415 & 0.004 \\
\hline $35-39$ & 220 & 741 & 0.008 & 266 & 859 & 0.009 \\
\hline $40-44$ & 364 & 1.395 & 0.015 & 456 & 1.496 & 0.016 \\
\hline $45-49$ & 421 & 1.697 & 0.018 & 554 & 1.751 & 0.018 \\
\hline $50-54$ & 394 & 1.690 & 0.018 & 626 & 2.058 & 0.022 \\
\hline $55-59$ & 367 & 1.509 & 0.016 & 606 & 1.893 & 0.020 \\
\hline $60-64$ & 302 & 1.216 & 0.013 & 447 & 1.452 & 0.015 \\
\hline $65-69$ & 274 & 1.170 & 0.012 & 388 & 1.346 & 0.014 \\
\hline $70-74$ & 231 & 992 & 0.010 & 328 & 1.065 & 0.011 \\
\hline $75-79$ & 160 & 373 & 0.004 & 283 & 463 & 0.005 \\
\hline Total & 2.910 & 11.446 & 0.120 & 4.119 & 12.950 & 0.136 \\
\hline \multicolumn{7}{|c|}{ Cáncer cérvico uterino } \\
\hline \multirow{2}{*}{$\begin{array}{l}\text { Grupo } \\
\text { de edad }\end{array}$} & \multicolumn{3}{|c|}{1997} & \multicolumn{3}{|c|}{2007} \\
\hline & Defunciones & AVP & $\begin{array}{c}\text { Promedio } \\
\text { de AVP }\end{array}$ & Defunciones & AVP & $\begin{array}{c}\text { Promedio } \\
\text { de AVP }\end{array}$ \\
\hline $20-24$ & 9 & 30 & 0.000 & 12 & 36 & 0.000 \\
\hline $25-29$ & 56 & 209 & 0.002 & 49 & 165 & 0.002 \\
\hline $30-34$ & 147 & 555 & 0.006 & 115 & 401 & 0.004 \\
\hline $35-39$ & 302 & 1.017 & 0.011 & 209 & 675 & 0.007 \\
\hline $40-44$ & 450 & 1.725 & 0.018 & 332 & 1.089 & 0.011 \\
\hline $45-49$ & 461 & 1.859 & 0.019 & 429 & 1.356 & 0.014 \\
\hline $50-54$ & 493 & 2.114 & 0.022 & 481 & 1.581 & 0.017 \\
\hline $55-59$ & 445 & 1.829 & 0.019 & 408 & 1.275 & 0.013 \\
\hline $60-64$ & 459 & 1.848 & 0.019 & 426 & 1.384 & 0.015 \\
\hline $65-69$ & 444 & 1.896 & 0.020 & 402 & 1.395 & 0.015 \\
\hline $70-74$ & 445 & 1.911 & 0.020 & 359 & 1.165 & 0.012 \\
\hline $75-79$ & 300 & 700 & 0.007 & 337 & 552 & 0.006 \\
\hline Total & 4.011 & 15.694 & 0.164 & 3.559 & 11.073 & 0.116 \\
\hline
\end{tabular}

Fuente: Elaboración propia a partir de información de las Estadísticas Vitales de Mortalidad del INEGI para 1997 y 


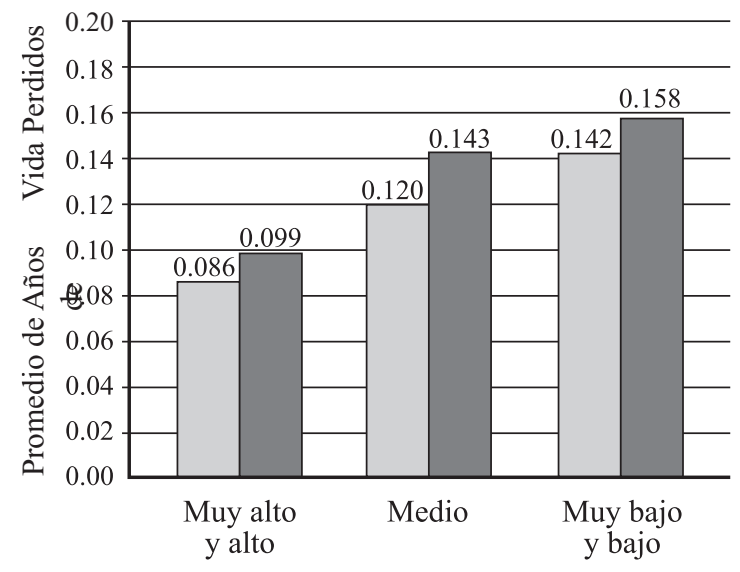

Grado de Marginación

\begin{tabular}{|l}
$\square$ \\
$\square$
\end{tabular} 2007

Fuente: Elaboración propia a partir de información de las Estadísticas Vitales de Mortalidad del INEGI para 1997 y 2007.

Figura 1. Promedio de años de vida perdidos por cáncer de mama según grado de marginación estatal. México, 1997 y 2007.

emprender acciones de información a las mujeres sobre estas dos afecciones, lo que podría tener un impacto altamente positivo en el cuidado autopersonal ${ }^{22-24}$. Un aspecto fundamental es lograr que las mujeres sepan que tanto el CaMa como el $\mathrm{CaCu}$ son enfermedades prevenibles y curables si son identificadas oportunamente ${ }^{25-27}$.

Aunque los datos muestran que los AVP debidos al $\mathrm{CaCu}$ han disminuido considerablemente entre 1997 y 2007, es indispensable seguir reforzando acciones dirigidas a mitigar su impacto, así como también es imperante llevar a cabo una vigilancia permanente al CaMa. Las diferencias socioeconómicas, de acuerdo con el nivel de marginación de los estados, ponen de manifiesto que en México existe una variedad de panoramas epidemiológicos $7,11,13,15,28$, en donde se traslapan múltiples estilos y condicionantes de vida que requieren de distintos tipos de recursos e intervenciones ${ }^{7,15}$. Las tendencias en la mortalidad por $\mathrm{CaCu}$ y $\mathrm{CaMa}$, así como los AVP observados en este trabajo coinciden con resultados encontrados por otros expertos en el tema y en otros contextos latinoamericanos, en donde se muestra que el CaMa está superando los niveles alcanzados por el $\mathrm{CaCu}^{13,29}$.
Concomitante con este último padecimiento mencionado, se ha discutido en otras investigaciones que las mujeres que habitan en áreas menos desarrolladas se encuentran con mayores obstáculos asociados con el acceso a los sistemas de salud, la atención oportuna, el trato del personal de salud, los tabúes culturales, la capacidad de pago de servicios de salud (consultas, medicamentos y tratamientos), entre otros. Aquellas que viven en áreas rurales o marginadas se enfrentan a dificultades geográficas para desplazarse a los centros de salud y quienes pertenecen a determinados grupos étnicos (como afrodescendientes e indígenas) sufren discriminación o tienen limitada capacidad de comunicación, por lo que sus posibilidades de sobrevivencia disminuyen significativamente ${ }^{11-14,29-32}$.

Los resultados obtenidos para CaMa se asemejan a los de otros trabajos donde se ha demostrado que existe un mayor riesgo de morir por esta causa en regiones más desarrolladas ${ }^{29,33,34}$. En el caso de América Latina -al igual que en este estudio- las tendencias de la mortalidad siguen un patrón ascendente para diversos grupos diferenciados socioeconómicamente ${ }^{29}$, entre tanto, un amplio sector académico ha subrayado que las mujeres más pobres tienen reducidas sus capacidades de detección temprana de la enfermedad mediante técnicas de screening (autoexploración mamaria, examen clínico de mama y mamografía), provocando que en caso de diagnosticarse, el resultado sea el más desfavorable ${ }^{12,23,33,34}$.

Uno de los argumentos más fuertes que explica la caída de la mortalidad y, por ende, los AVP por $\mathrm{CaCu}$ en México, es la implementación de programas tendientes a la prevención y diagnóstico temprano de la tumoración, lo cual se ha reforzado con una permanente campaña de información a las mujeres y el personal de salud ${ }^{22}$; contrariamente, dado el carácter multicausal del $\mathrm{CaMa}^{35}$ aún no se conoce con precisión cuáles son los factores que están asociados con una mayor o menor variación en la distribución de las defunciones; algunos investigadores sostienen que esto es consecuencia, en parte, de una mayor 


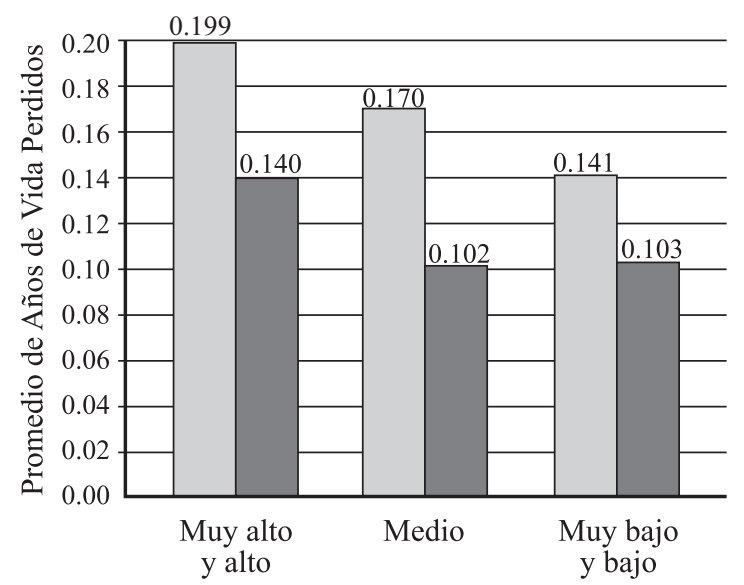

Grado de Marginación

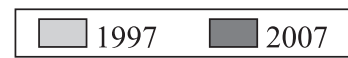

Fuente: Elaboración propia a partir de información de las Estadísticas Vitales de Mortalidad del INEGI para 1997 y 2007.

Figura 2. Promedio de años de vida perdidos por cáncer cérvico uterino según grado de marginación estatal. México, 1997 y 2007.

notificación de casos, especialmente entre las mujeres con mejor acceso a los servicios de salud lo que conlleva a una subestimación del efecto del CaMa en poblaciones vulnerables ${ }^{29}$.

En lo que toca a la estimación de los AVP se cree conveniente la inclusión de este tipo de análisis, dada las amplias posibilidades de medir cambios en los niveles y las tendencias de la mortalidad, especialmente por grupos de edad, con lo cual también se identifican las poblaciones más afectadas por las dos tumoraciones y en aquellas donde es necesario focalizar los programas y recursos con los que se cuenta.

La desagregación socioeconómica por su parte se utilizó para comprender de manera diferencial el comportamiento del CaMa y el $\mathrm{CaCu}$ en dos puntos del tiempo, evidenciándose la doble carga para la salud que implican las dos afecciones.

Finalmente es preciso decir que el aporte principal de este trabajo es el análisis empírico de un indicador que ha sido poco utilizado en el ámbito nacional y que suministra elementos de gran interés para el conocimiento de otra arista respecto a la mortalidad por estos dos cánceres en las mujeres mexicanas. Es por ello que entre las tareas que siguen pendientes está el mejoramiento permanente de los registros vitales (defunciones) y la adecuada clasificación de las causas de muertes, con el fin de que los cálculos se ajusten de manera más adecuada a la realidad.

\section{Limitaciones}

La principal limitación en el uso de los AVP es que se requiere acotar las edades bajo estudio y dejar por fuera del análisis a mujeres que integran un grupo abierto de edad ${ }^{4-5}$ (en este caso en concreto, de los 80 años y más). Por lo tanto, el indicador no incluye a todas las mujeres en riesgo de morir por estas causas. Asimismo, los AVP se calculan con datos provenientes de estadísticas vitales, que presentan ciertas restricciones como: falta de uniformidad en las definiciones del registro, cobertura inadecuada del Registro Civil en regiones del país poco accesibles, errores de omisión, subregistro de las defunciones, entre otras ${ }^{36}$.

Otra dificultad se deriva de la información de los registros de mortalidad, ya que con esta fuente no es posible conocer el nivel socioeconómico de las fallecidas, por lo que el grado de marginación al que se refiere este estudio corresponde al lugar de residencia habitual que se declara en las estadísticas vitales de mortalidad, sin que esta atribución concierna con una estricta clasificación de las mujeres. Esto significa que en cada uno de los estratos se tiene tanto población pobre como no pobre, por lo que resultaría erróneo interpretar a los estratos bajos como los estratos pobres o asociar a la población que concentran como una medida de pobreza. Lo que sí es importante rescatar, es que es mucho más probable encontrar población clasificada como pobre en los estratos bajos que en los altos, por lo que los resultados obtenidos a partir de esta estratificación se pueden utilizar para efectos de focalización de políticas públicas ${ }^{37}$.

\section{REFERENCIAS}

1. BERNABEU J. Enfermedad y población. Valencia: Universitat d'Alacant; 1991. 
2. CORONA R, JIMÉNEZ R. El comportamiento de la mortalidad por entidad federativa, 1980. México: Universidad Autónoma de México; 1988.

3. GARCÍA C. Causas de muerte como indicadores indirectos de necesidades en salud. Una interpretación sociodemográfica. En: García C, Hernández H, coordinadores. Mortalidad, salud y discurso demográfico. México: Universidad Nacional Autónoma de México/Centro Regional de Investigaciones Multidisciplinarias; 1996. p. 167-203.

4. ARRIAGA E. Comentarios sobre algunos índices para medir el nivel y el cambio de la mortalidad. Estudios Demográficos y Urbanos 1996; 11:5-30.

5. ARRIAGA E. Los años de vida perdidos: Su utilización para medir el nivel y cambio de la mortalidad. Notas de Población 1996; 24:7-38.

6. BOCCO M. La relación entre los años de vida perdidos y la esperanza de vida: Aplicaciones para el análisis de la mortalidad. Notas de Población 1996; 24; 3960.

7. FRENK J. Transiciones, vidas, instituciones, ideas. Salud Pública de México 1997; 39:144-150.

8. INSTITUTO NACIONAL DE ESTADÍSTICA, GEOGRAFÍA E INFORMÁTICA (INEGI). Mujeres y Hombres en México 2009. México: Instituto Nacional de Estadística, Geografía e Informática; 2009.

9. Secretaria de Salud de México/Sistema de Información Nacional en Salud. Estadísticas de Mortalidad [serial online] 2010 [citado 12 Ene 2010]; [1 pantalla]. Disponible en: URL: www.sinais.salud.gob.mx/mortalidad/index.html

10. SECRETARÍA DE SALUD DE MÉXICO. Programa Nacional de Salud 2007-2012. México: Secretaría de Salud de México.

11. PALACIO LS, LAZCANO E, ALLEN B, HERNÁNDEZ M. Diferencias regionales en la mortalidad por cáncer de mama y cérvix en México entre 1979 y 2006. Salud Pública de México 2009; 51:208-218

12. AGUDELO M, PALACIO LS. Prácticas de detección de cáncer de mama en mujeres de áreas marginales del Distrito Federal. 2010; Mimeo.

13. MARTÍNEZ C, MARTÍNEZ RM. La mortalidad por cáncer cérvicouterino y de mama en Colombia y México como expresión de las desigualdades socioeconómicas. En: Rodríguez L, organizador. Población y salud sexual y reproductiva en América Latina; 2008.p.303-327.

14. SOSA S, WALKER D, SERVÁN E. Práctica de mastografías y pruebas de papanicolau entre mujeres de áreas rurales de México. Salud Pública de México 2009; 51:236-245.

15. FRENK J, BOBADILLA JL, STERN C, FREJKA T, LOZANO R. Elementos para una teoría de la transición en salud. Salud Pública de México 1991; 33:448-462.

16. ORGANIZACIÓN PANAMERICANA DE LA SALUD (OPS)/Oficina Sanitaria Panamericana/Oficina Regional de la Organización Mundial de la salud (OMS). Clasificación Estadística Internacional de
Enfermedades. 9a revisión. Washington, DC: Organización Panamericana de la Salud; 1994.

17. ORGANIZACIÓN PANAMERICANA DE LA SALUD (OPS)/Oficina Sanitaria Panamericana/Oficina Regional de la Organización Mundial de la salud (OMS). Clasificación Estadística Internacional de Enfermedades y Problemas Relacionados con la Salud. $10^{\mathrm{a}}$ revisión. Washington, DC: Organización Panamericana de la Salud; 2003.

18. MINA, A. Funciones de sobrevivencia empleadas en el análisis demográfico. Papeles de Población 2001; 28:131-154.

19. CONSEJO NACIONAL DE POBLACIÓN (CONAPO). Índice de marginación 2005. México: Consejo Nacional de Población.

20. KNAUL F, LÓPEZ L, AGUILAR B, ALTAHUS E, ARREOLA H, BARRERIRO N, et al. Reporte Rosa 2009-2010: “Cáncer de mama a con-ciencia” México: Fundación Mexicana para la Salud; 2009.

21. KNAUL F, BUSTREO F, HA E, LANGER A. Breast cáncer: Why link early detection to reproductive health interventions in developing countries? Salud Pública de México 2009; 51:220-227.

22. NIGENDA G, CABALLERO M, GONZÁLEZ LM. Barreras en el acceso al diagnóstico temprano del cáncer de mama en el Distrito Federal y en Oaxaca. Salud Pública de México 2009; 51:254-262.

23. LÓPEZ L, TORRES L, LÓPEZ M, RUEDA C. Identificación del lesiones mamarias malignas en México. Salud Pública de México 2001; 43:199-202.

24. ALIANZA PARA LA PREVENCIÓN DE CÁNCER CERVICAL (ACCP). Prevención del cáncer cervicouterino. Ficha descriptiva. Washington: Alianza para la Prevención de Cáncer Cervical.

25. TREJO U, GRANADOS JA, ORTIZ L, DELGADO G. Diferencias sociales de la detección oportuna de cáncer cérvico uterino en las mujeres trabajadoras de una universidad de la Ciudad de México. Revista Española de Salud Pública 2005; 79:403-414.

26. MARTÍNEZ O, URIBE P, HERNÁNDEZ M. Políticas públicas para la detección del cáncer de mama en México. Salud Pública de México 2009; 51:350-360.

27. ANAND P, KUNNUMAKARA A, SUNDARAM C, HARIKUMA KB, THARAKAN S, LAI O, et al. Cancer is a Preventable Disease that Requires Major Lifestyle Changes. Pharmaceutical Research 2008; 25:2097-2116.

28. LOZANO C, ZÚÑIGA IR. Perfil epidemiológico del cáncer cervicouterino en México. Revista de Enfermedades Infecciosas en Pediatría 2009; 90: 3637.

29. LOZANO R, GÓMEZ H, LEWIS S, TORRES L, LÓPEZ L. Tendencias del cáncer de mama en América Latina y El Caribe. Salud Pública de México 2009; 51:147-156.

30. CUTHBERTSON SA, GOYDER EC, POOLE J. Inequalities in breast cancer stage at diagnosis in the trent region, and implications for the NHS Breast Screening Programme. Journal Public Health 2009; 31:398-405. 
31. BHAREL M, CASEY C, WITTENBERG E. Disparities in cancer screening: acceptance of Pap smears among homeless women. Journal Womens Health 2009; 18: 2011-2016.

32. CASI A, MORENO C. Nivel socioeconómico y mortalidad. Rev San Hig Pub 1992; 66: 17-28.

33. SMITH R, CALEFFI M, ALBERT US, CHEN T, DUFFY S, FRANCESCHI D, et al. El cáncer de mama en países de recursos limitados: Detección temprana y acceso a la asistencia. The Breast Journal 2007; 13 (1):16-29.

34. ANDERSON B, YIP CH, RAMSEY S, BENGOA R, BRAUN S, FITCH M, et al. El cáncer de mama en países de recursos limitados: Sistemas de atención de salud y políticas públicas. The Breast Journal 2007; 13 (1):62-82.
35. GONZÁLEZ M, CHÁVEZ F, ROMERO T, LENCE J. Riesgo de morir por cáncer de mama en Cuba. Rev Cubana Oncol 1998; 141(1):22-25.

36. LOZANO, R. ¿Es posible seguir mejorando los registros de las defunciones en México? Gac Med Mex 2008; 144 (6): 525-534.

37. INSTITUTO NACIONAL DE ESTADÍSTICA Y GEOGRAFÍA (INEGI). Regiones socioeconómicas de México, 2002 [online] 2010 [citado 20 Jul 2010]; [79 pag.]. Disponible en: http://jweb.inegi.gob.mx/niveles/datosnbi/reg_soc_ mexico.pdf.

Recepción: 16 de abril de 2010 Aprobación: 26 julio de 2010

Usted puede comentar éste y otros artículos publicados en la Revista Chilena de Salud Pública, enviando un correo electrónico a revistasp@med.uchile.cl 